\title{
ARTIGO DE ATUALIzAçÃo Resiliência e transtorno do déficit de atenção/hiperatividade
}

Resilience and attention deficit/hyperactivity disorder

Maria Angélica Regalla', Priscilla Rodrigues Guilherme', Maria Antônia Serra-Pinheiro

\section{RESUMO}

Este artigo tem como objetivo fazer uma atualização acerca dos conceitos de resiliência, fatores de risco e fatores de proteção, correlacionando-os com o impacto do transtorno do déficit de atenção/hiperatividade (TDAH). Resiliência descreve a capacidade do indivíduo de, uma vez exposto a estresse, resistir e vencê-lo. A família está envolvida no conceito de resiliência, tanto pela sua capacidade de interferir na resiliência dos seus indivíduos componentes quanto na habilidade de responder como uma unidade funcional resiliente, diante do estresse. O relacionamento com figuras afetivamente importantes na infância, o número pequeno de estressores, e o entendimento subjetivo em relação ao estressor são fatores que influenciam a capacidade de ser resiliente. A ausência de comorbidades, de problemas de conduta ou de relacionamento com colegas, bem como de sintomas somáticos ou problemas de coordenação, está associada com menor impacto negativo do TDAH.

\section{ABSTRACT}

The aim of this article is to perform an update regarding the definition of resilience, risk factors and protective factors, and to correlate them with the impact of Attention Deficit/Hyperactivity Disorder (ADHD). Resilience is the capacity to resist and overcome adversity. Family is associated with the concept of resilience not only because of its capacity to interfere with the resilience of its members, but also because of its ability to respond as a functional unit when challenged with adversity. Relationships with affectively meaningful figures during childhood, the number of adverse events to which the individual is submitted and his subjective understanding of the stressor modify the ability to be resilient. ADHD has a smaller negative impact in children without conduct problems, social relationship problems, somatic symptoms or coordination problems.

\section{Key-words}

Resilience, attention deficit/ hyperactivity disorder, risck factors, protection factors.

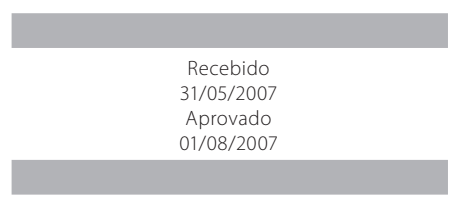

1 Instituto de Psiquiatria da Universidade Federal do Rio de Janeiro (IPUB-UFRJ).

Endereço para correspondência: Maria Angélica Regalla

Estrada do Galeão, 961/202 - 21931-000 - Ilha do Governador, RJ

Fone: (21) 3353-3697 -E-mail: angelica.regalla@uol.com.br 


\section{INTRODUÇÃO}

Resiliência pode ser definida como um processo ativo de resistência, reestruturação e crescimento em resposta à crise e ao desafio (Walsh, 1998). O conceito de resiliência vem sendo bastante discutido do ponto de vista teórico e metodológico pela comunidade científica, principalmente por tratar-se de um conceito relativamente novo no campo da psicologia. Na década de 1970, o conceito foi estudado em pesquisas com pessoas que foram submetidas a estresses agudos ou persistentes e não adoeceram como seria o esperado.

O TDAH é o transtorno psiquiátrico mais comum na infância, acometendo cerca de 3\% a 6\% das crianças desde tenra idade (Rohde et al., 1999). Diversos estudos evidenciaram a persistência do transtorno do déficit de atenção/hiperatividade na vida adulta (Rohde et al., 2003) e seu importante impacto sobre a vida pessoal, social, familiar e acadêmica (Rohde et al., 1999) dos portadores desse transtorno. No entanto, poucos estudos procuraram descrever características dos indivíduos acometidos e de seu ambiente, que possam agir como fatores ou mecanismos protetores e influenciar no desenvolvimento de resiliência.

O presente trabalho pretende apresentar algumas definições sobre o conceito de resiliência, discutir os mecanismos de proteção e os fatores de risco, abordar aspectos familiares da resiliência, assim como as possíveis correlações desse conceito com o transtorno do déficit de atenção/hiperatividade.

\section{O conceito de resiliência}

A resiliência é um conceito que tem sido explorado e aplicado nas mais diversas áreas que envolvem o ser humano e seu ambiente de relacionamento. Com origem nas ciências físicas, a utilização do conceito nas ciências da saúde acontece na década de 1970, com estudos sobre pessoas que, mesmo tendo sido submetidas a traumas agudos ou prolongados, não adoeciam como seria o esperado.

O conceito de resiliência foi definido inicialmente como um conjunto de traços de personalidade e capacidades que tornavam invulneráveis as pessoas que passavam por experiências traumáticas e não desenvolviam doenças psíquicas, caracterizando, assim, a qualidade de serem resistentes (Anthony e Cohler, 1987).

Posteriormente, Fonagy et al. (1994) atribuíram grande importância ao relacionamento com figuras de apego na infância como parte do processo de recuperação e desenvolvimento saudável. Considerando-se, portanto, a possibilidade do desenvolvimento da resiliência, não só pelas características de personalidade como também por meio do relacionamento com pessoas significativas.
Rutter (1987) acrescenta que a resiliência não deve ser vista como um atributo fixo do indivíduo, de forma que, se as circunstâncias relacionadas a esse indivíduo mudam, a resiliência se altera. Os resultados de suas pesquisas originaram a afirmação de que um único estressor pode não ter impacto significativo, mas que a combinação de dois ou mais estressores diminui a possibilidade de conseqüências positivas no desenvolvimento, ou seja, estressores adicionais aumentam o impacto de outros estressores já presentes na vida do indivíduo.

A resiliência se dá a partir de processos de proteção que englobam quatro principais funções: (1) reduzir o impacto dos riscos; (2) reduzir as reações negativas em cadeia que seguem a exposição do indivíduo à situação de risco; (3) estabelecer e manter a auto-estima e auto-eficácia, por meio de relações de apego seguras e o cumprimento de tarefas com sucesso; (4) criar oportunidades para reverter os efeitos do estresse (Rutter, 1987).

O crescente interesse pelo conceito de resiliência, reflexo da necessidade de prevenção de problemas e promoção da saúde mental, gera esforços para a construção de instrumentos de medida da qualidade de ser resiliente. A escala de resiliência desenvolvida por Wagnild e Young (1993) é um dos poucos instrumentos usados para medir níveis de adaptação psicossocial, e estudos iniciais obtiveram bons indicativos de confiabilidade e validade desse instrumento. No Brasil, essa escala foi adaptada e validada por Pesce et al. (2005), em uma amostra de escolares dos ensinos fundamental e médio da rede pública de ensino do município de São Gonçalo, Rio de Janeiro.

\section{Resiliência e família}

O conceito de resiliência se associa à família de duas formas: há estudos que investigam a capacidade de famílias reagirem à adversidade de forma funcional e há investigações acerca do papel desempenhado pela família para desenvolvimento da resiliência do indivíduo.

Um dos primeiros trabalhos específicos de resiliência com foco na família foi publicado por McCubbin e McCubbin (1988) sobre a "tipologia de famílias resilientes", partindo da definição segundo a qual famílias resilientes são aquelas que resistem aos problemas decorrentes de mudanças e adaptam-se às situações de crise. Essa adaptação ultrapassa o simples ajustamento, pois envolve a mudança de crenças e de visão de mundo. Esse processo passaria pelos recursos internos da família e os externos da comunidade, considerando-se, ainda, o fator espiritual. Walsh (1996) organiza seus conhecimentos na área da resiliência familiar e propõe um panorama conceitual dentro de três domínios: sistema de crenças da família (atribuir sentido à adversidade, olhar positivo, transcendência e espiritualidade), padrões de organização (flexibilidade, coesão, recursos sociais e econômicos) e processos de comunicação (clareza, expres- 
sões emocionais, colaboração na resolução de problemas). Como ilustração desse modelo, observa-se que famílias de migrantes, que se mostram resilientes, são aquelas mais estáveis e integradas (Greeff e Holtzkamp, 2007).

Com relação à família como fator promovedor de resiliência no indivíduo, Fonagy et al. (1994) já haviam apontado para centralidade de figuras de apego no desenvolvimento de resiliência. A estrutura familiar é um fator importante de variação no risco de desenvolvimento de uma série de condições, tais como comportamento disruptivo (McGee et al., 2002) e faltas escolares não-médicas (Miller et al., 1999). A percepção de suporte familiar se associa com maior resiliência em indivíduos sujeitos a inúmeros estressores (Li et al., 2007).

\section{Fatores de risco e fatores de proteção}

Considerando-se que as experiências de vida negativas são inevitáveis para qualquer indivíduo, sobressai a questão dos níveis de exposição e dos limites individuais. Assim, a visão subjetiva de um indivíduo a determinada situação, ou seja, sua percepção, interpretação e sentido atribuído ao evento estressor, é que interferirá na sua classificação ou não como condição de estresse. Por essa razão, um evento pode ser avaliado como perigo por um indivíduo e para outro ser apenas um desafio (Yunes e Szymanski, 2001). Eventos considerados como risco são obstáculos individuais ou ambientais que aumentariam a vulnerabilidade da criança e do adolescente para resultados negativos no seu desenvolvimento (Engle et al., 1996).

Entre os indicadores de risco que se constituem em dificuldades e problemas para a família, Antoni e Koller (2000) relacionaram descontrole emocional, culpa, falta de responsabilidade e de diálogo, drogas, ausência dos pais, violência doméstica e na comunidade, transição da infância para a adolescência e ausência das redes de apoio.

Resultados de estudos restropectivos com pessoas consideradas resilientes apontaram para a importância de crenças e significados, esperança, espiritualidade e sentido de vida, aliado à visão otimista de futuro, como fatores que influenciam no processo de avaliação dos eventos estressores, de busca de recursos e do aprendizado com a experiência (McCubbin et al., 1996; Walsh, 1996, 1998). O otimismo (Li et al., 2007) já se mostrou como fator de resiliência em populações de risco.

Altos níveis de inteligência, baixos níveis de comportamento anti-social do indivíduo e dos pais aumentam a capacidade de ser resiliente (Jaffee et al., 2007). Da mesma forma, vínculos sociais e competência pessoal se associam com maior resiliência (Lussier et al., 2007). Suporte da comunidade também aumenta a resiliência (Greeff e Holtzkamp, 2007).

\section{Resiliência e TDAH}

As características nucleares do TDAH são a desatenção, a hiperatividade e a impulsividade. Elas afetam de modo adverso o desempenho acadêmico (Rohde et al., 1999), os relacionamentos familiar e social (Barkley, 1998), o ajustamento psicossocial e a vida laborativa. Além dos sintomas básicos do transtorno, em mais de $50 \%$ dos casos, existem comorbidades com transtornos do aprendizado, transtornos do humor e de ansiedade, transtornos disruptivos do comportamento e transtornos do abuso de substâncias e de álcool (Biederman et al., 1993). Além disso, crianças que experimentam o fracasso precocemente, algo comum no TDAH, são vulneráveis a uma ampla variedade de complicações psicossociais. Essas complicações, por sua vez, podem levar a problemas crônicos em diferentes esferas da vida, persistindo mesmo quando os sintomas nucleares do transtorno remitiram ou melhoraram de modo significativo ao longo dos anos (Rohde e Mattos, 2003).

O impacto do TDAH, a longo prazo, na vida das crianças não é uniforme. Cerca de $20 \%$ das crianças com TDAH persistente têm bom funcionamento em várias áreas de sua vida, enquanto $20 \%$ das crianças com TDAH persistente funcionam mal em âmbitos social, educacional e emocional (Biederman et al., 1998). Essa variabilidade de adaptação levanta a importância da resiliência no TDAH.

Riley et al. (2006) observaram que crianças com TDAH e sem problemas de conduta ou de relacionamento com colegas, sem sintomas somáticos ou problemas de coordenação, tinham qualidade de vida melhor, de forma que inúmeros fatores influenciavam o impacto do TDAH na vida da criança. Problemas psiquiátricos na mãe, impulsividade, comorbidades psiquiátricas e famílias com numerosos fiIhos podem diminuir a chance de normalização no funcionamento das crianças portadoras de TDAH (Biederman et al., 1998), indicando, assim, baixa resiliência.

Sabe-se que rejeição por pares é um fator de risco para o desenvolvimento de problemas na vida adolescente e adulta (Parker e Asher, 1987). É notório, também, que TDAH está associado com rejeição por pares (Miller-Johnson et al., 2002). Assim sendo, Mikami e Hinshaw (2006) investigaram fatores associados com resiliência em meninas, de 6 a 12 anos, com ou sem diagnóstico para TDAH, que eram rejeitadas por seus pares. Evidenciaram que rejeição por pares foi associada a altos níveis de comportamento agressivo e sintomas de ansiedade e depressão. Popularidade com adultos, para todas as meninas estudadas, predisse baixos níveis de agressividade, assim como envolvimento em brincadeiras solitárias com metas foi associado com baixos níveis de ansiedade e depressão, especialmente em crianças rejeitadas por seus pares. $\mathrm{O}$ estudo apontou que algumas meninas que pertenciam ao subgrupo que eram rejeitadas por seus pares, portanto, possuíam características (popula- 
ridade com adultos e a capacidade de brincarem sozinhas envolvidas com metas) que poderiam contribuir para serem resilientes.

Mikami e Hinshaw (2006), em estudo longitudinal com adolescentes, selecionaram três hipotéticos fatores de proteção (autopercepção de competências escolares, envolvimento em brincadeiras com metas e popularidade com adultos, que não os pais) para avaliação de resiliência em meninas com TDAH. Essas hipóteses foram escolhidas porque crianças com TDAH, rejeitadas por seus pares, gastam um tempo considerável sozinhas e experimentam uma grande proporção de fracassos acadêmicos e sociais na escola. Logo, esses fatores protetores forneceriam para a criança distração e envolvimento quando estão isoladas na escola. O estudo revelou que tanto rejeição por pares quanto TDAH na infância predizem uma ampla gama de resultados negativos na adolescência, como comportamentos externalizantes, comportamentos internalizantes, maus resultados acadêmicos, transtornos alimentares e uso de substância (somente com diagnóstico de TDAH). Ao contrário da hipótese original, o envolvimento em brincadeiras com metas foi consistentemente associado com maior incidência de transtornos psiquiátricos na adolescência, particularmente entre meninas com TDAH. Além disso, a variável "popularidade com adultos" não se apresentou como fator de proteção contra problemas no comportamento adolescente. Surpreendentemente, os resultados apontaram fraca interação entre os fatores de proteção, inicialmente associados com resiliência, e TDAH. Porém, a autopercepção de competências escolares protegeu contra o surgimento de comportamentos internalizantes, comportamentos externalizantes e uso de substâncias.

Um dos mais discutidos riscos associados ao TDAH é o de uso de drogas ilícitas. Wilens et al. (2003) demonstraram que crianças medicadas para TDAH tinham menor risco de desenvolver uso de drogas. Dessa forma, o uso de medicação poderia aumentar a capacidade da criança de lidar de forma positiva com o estressor, no caso, o TDAH. Existem diversos fatores que se associam, por sua vez, à chance de ser medicado, dentre eles, ser atendido por um profissional de saúde mental, região geográfica de residência e fatores sociodemográficos (Radigan et al., 2005).

A família é um importante contexto para que a criança aprenda a se relacionar com o mundo. Famílias ensinam às crianças como se aproximar das pessoas, cuidar dos relacionamentos, resolver problemas e traçar metas, assim como negociar as tensões entre desejos individuais e expectativas sociais (Kendall, 1998). Estes são desafios para todas as crianças, especialmente para as crianças com TDAH.

Existem fortes evidências de que o ajustamento emocional e comportamental da criança com TDAH sofre impacto significativo de fatores ambientais, tanto quanto por fatores genéticos, sendo a família a mais importante provedora do ambiente social da criança (Hechtman, 1996).

Chronis et al. (2007) evidenciaram que "hábitos educacionais positivos" empregados pelos pais em crianças com TDAH diminuíam o risco de essas crianças posteriormente desenvolverem problemas de conduta. Riley et al. (2006) evidenciaram que problemas de saúde nos pais e não morar com os dois pais diminuíam a qualidade de vida da criança com TDAH, sugerindo facilitar um maior impacto do TDAH sobre as crianças e, portanto, menor resiliência.

Além da questão da resiliência em pacientes com TDAH, existe a questão da resiliência em pacientes com fatores de risco para o desenvolvimento de TDAH. Que crianças serão menos suscetíveis ao desenvolvimento do transtorno, dada sua maior vulnerabilidade genética? Martell e Nigg (2006) evidenciaram que crianças com dificuldades de auto-regulação estavam especialmente sujeitas ao risco de desenvolvimento de TDAH.

\section{CONCLUSÕES}

Resiliência é a habilidade de lidar com a adversidade sem sucumbir ou ser aleijado por ela. Classicamente, o modelo médico trabalha com fatores de risco e proteção: marcadores que aumentam ou diminuem o risco de um determinado desfecho. O conceito de resiliência é mais elástico e incorpora os conceitos de fatores de risco e proteção de uma forma mais dinâmica, interativa e pessoal.

TDAH é uma condição associada freqüentemente com diversos desfechos desfavoráveis. Essa associação é determinada pelo fato de pacientes com TDAH terem muito maior chance de chegarem a esses desfechos do que controles sem TDAH. Pacientes com TDAH têm maiores índices de reprovações escolares (Rohde et al., 1999), maiores índices de uso de substâncias (McGee et al., 2002) e maiores índices de problemas sociais (Barkley, 1998). Muitos sujeitos com TDAH, no entanto, não evoluem para esses desfechos negativos. É essencial descobrir o que faz o sujeito menos suscetível à adversidade, aqui representada pelo TDAH.

Muito pouco ainda se sabe sobre o que torna o indivíduo com TDAH menos vulnerável. A ausência de comorbidades parece diminuir o risco de uso de drogas em pacientes com TDAH (McGee et al., 2002). Estruturação familiar parece diminuir o risco de disfunções emocionais e de comprometimento de qualidade de vida (Riley et al., 2006). Características como avaliação positiva da vida e boas habilidades comunicativas (Walsh, 1996) são associadas com indivíduos resilientes, independentemente da presença de TDAH. É provável que sejam associadas à diminuição do risco de desfechos ruins também no caso de pacientes com TDAH. Muito ainda precisa se saber em relação aos mecanismos 
e às características que fazem com que o TDAH tenha um impacto menos negativo sobre a vida dos pacientes a que ele acomete.

Os resultados de pesquisas investigando fatores de resiliência podem ser usados para o desenvolvimento e promoção de resiliência, com portadores de TDAH, no tratamento do transtorno e na orientação de suas famílias.

Potenciais conflitos de interesse: $O$ GEDA - UFRJ recebe suporte de pesquisa do Laboratório Janssen-Cilag.

\section{REFERÊNCIAS}

Anthony EJ, Cohler BJ. The invulnerable child. New York: Guilford Press; 1987.

Antoni C, Koller S. Vulnerabilidade e resiliência familiar: um estudo com adolescentes que sofreram maus-tratos intrafamiliares. Rev Psic, 31(1):39-66, 2000

Barkley RA. Attention déficit hyperacyivity disorder; a handbook for diagnosis and treatment. New York: Guilford; 1998.

Biederman J, Faraone SV, Spencer T, Wilens T, Norman D, Lapey KA et al. Patterns of psychiatric comorbidity, cognition and psychosocial functioning in adults with attention deficit disorder. Am J Psychiatry, 150:1792-8, 1993

Biederman J, Faraone SV, Taylor A, Sienna M, Williamson S, Fine C. Diagnostic continuity between child and adolescent ADHD: findings from a longitudinal clinical sample. J Am Ac Child Adolesc Psychiatry, 37(3):305-13,1998.

Chronis AM, Lahey BB, Pelham WE Jr, Williams SH, Baumann BL, Kipp H et al. Maternal depression and early positive parenting predict future conduct problems in young children with attention-deficit/hyperactivity disorder. Dev Psychol, 43(1):70-82, 2007.

Engle PI, Castle S, Menon P. Child development: vulnerability and resilience. Soc Scie Med, 43(5):621-35, 1996.

Fonagy P, Steele M, Steele H, Higgitt A, Target M. The Emanuci Miller Memorial Lecture 1992: the theory and practice of resilience. J Child Psychol Psychiatry, 35(2):231-57, 1994.

Greeff AP, Holtzkamp J. The prevalence of resilience in migrant families. Fam Com Health, 30(3):189-200, 2007

Hechtman L. Families of children with attention deficit/hyperactivity disorder: a review. Can J Psychiat, 42(6):350-360, 1996

Jaffee SR, Caspi A, Moffitt TE, Polo-Tomã SM, Taylor A. Individual, family and neighborhood factors distinguish resilient from non resilient maltreated children: a comulative stressor model. Child Abuse Negl, 31(3):231-53, 2007.

Kendall J. Outlasting disruption: The process of reinvestment in families with ADHD children. Qualitative Health Res, 8(6):839-57, 1998
Li ST, Richards MH. Risk and protective factors for urban African-American youth. Am J Com Psychol, 39:21-35, 2007

Lussier I, DerevenskyJL, Gupta R, Bervegin T, Ellenbogen S. Youth Gambling behaviors: an examination of the role of resilience. Psychol Addict Behav, 21(2):165-73, 2007.

Martell MM, Nigg TJ. Child ADHD and personality/temperament traits of reactive and effortful control, resiliency and emotionality. J Child Psychol Psychiat, 47(11):1175-83, 2006.

McCubbin HI, McCubbin MA. Typologies of resilient families: emerging roles of social class and ethnicity. Fam Relat, 37:247-54, 1988.

McGee R, Prior M, Williams S, Smart D, Sanson A. The long-term significance of teacher-rated hyperactivity and reading ability in childhood: findings from two longitudinal studies. J Child Psychol Psychiat, 43(8):1004-17, 2002

Mikami AY, Hinshaw SP. Resilient adolescent adjustment among girls: buffers of childhood peer rejection and attention deficit/hyperactivity disorder. J Abnormal Child Psychol, 34:825-39, 2006.

Miller P, Plant M. Truancy and perceived school performance: an alcohol and drug study of UK teenagers. Alcohol Alcohol, 34(6):886-9, 1999

Miller-Johnson S, Coie JD, Maumary-Gremaud A, Bierman KL. Conduct Problens Prevention Research Group. Peer rejection and aggression and early starter models of conduct disorder. J Abnormal Child Psychol, 30:217-30, 2002.

Parker JG, Asher SR. Peer relations and later personal adjustment: are low-accepted children at risk? Psychological Buletim, 102:357-89, 1987.

Pesce RP, Assis SG, Avanci JQ, Santos NC, Malaquias JV, Carvalhaes R. Adaptação transcultural, confiabilidade e validade da escala de resiliência. Cad Sau Publ, 21(2):436-48, 2005

Radigan M, Lannon P, Roohan P, Gesten F. Medication patterns for attention-deficit/hyperactivity disorder and comorbid psychiatric conditions in a low-income population. J Child Adolesc Psychopharmacol, 15(1):44-56, 2005

Riley AW, Spiel G, Coghill D, Dopfner M, Falissard B, Lorenzo MJ et al. ADORE Study Group. Factors related to Health-Related Quality of Life ( $\mathrm{HRQOL}$ ) among children with ADHD in Europe at entry into treatment. Eur Child Adolesc Psychiat, 15(1):38-45, 2006.

Rohde LA et al. ADHD in a school sample of Brazilian adolescents: a study of prevalence, comorbid conditions and impairments. J Am Acad Child Adolesc Psychiat, 38:716-22, 1999.

Rohde LA, Mattos P. Princípios e práticas em transtorno de déficit de atenção/hiperatividade. Porto Alegre: Artmed; 2003.

Rutter M. Psychosocial resilience and protective mechanisms. Am J Orthopsychiat, 57(3):316-31, 1987.

Wagnild GM, Young HM. Development and psychometric evaluation of resilience scale. J Nurs Meas, 1:165-78, 1993

Walsh F. The concept of family resilience: crisis and challenge. Family Process, 35:261-81, 1996

Walsh F. Strengthening family resilience. New York/London: The Guilford Press; 1998.

Wilens TE, Faraone SV, Biederman JG. Does stimulant therapy of attention deficit/hyperactivity disorder beget later substance abuse? A meta-analytic review of the literature. Pediatrics, 111(1):179-85, 2003

Yunes MAM, Szymanski H. Resiliência: noção, conceitos afins e considerações críticas. Em: Tavares J (org.) Resiliência e educação. São Paulo: Cortez; 2001, p. 13-42. 